\title{
Diet of Ornate Hawk-Eagle (Spizaetus ornatus)
}

\author{
Fagner Daniel Teixeira ${ }^{1,5}$, Elisa Paraíso Mesquita ${ }^{2}$ Michele Alves Ferreira $^{3} \&$ Felipe de Carvalho Araújo $^{4}$
}

\author{
1 Avenida João Gonçalves Teixeira, 22, Bairro Glória, Carmópolis de Minas, MG, Brazil. \\ 2 Rua Coronel Pedro Jorge, 26, Bairro Prado, Belo Horizonte, MG, Brazil. \\ 3 Rua Gustavo da Silveira, 1000, Bairro Horto Florestal, Belo Horizonte, MG, Brazil. \\ ${ }^{4}$ Departamento de Ciências Florestais, Universidade Federal de Lavras, Lavras, MG, Brazil. \\ 5 Corresponding author: fagnerdani@hotmail.com
}

Received on 12 November 2018. Accepted on 21 February 2019.

\begin{abstract}
The Ornate Hawk-Eagle (Spizaetus ornatus) is a top predator and inhabits mainly preserved forests. It occurs from Mexico to Argentina and throughout Brazil, where it is threatened by extinction. It hunts birds, mammals and reptiles, picking up both on the ground and on the branches in the forest. Here we report data on a pair and one young individual of this species registered in the southeast of Minas Gerais state, eastern portion of the Espinhaço Range, Brazil. In addition, a literature review on the diet of the species was carried out aiming gather data on food habits. The nesting territory, as well as the nest was discovered in semi-deciduous seasonal forest area. We recorded predation of a Lesser Yellow-headed Vulture (Cathartes burrovianus) by the young. After two days of observation, the nest was overthrown, what allowed its screening for other food items discovered after analysis of some feathers and bones. Detailed records of predation of S. ornatus were non-existent or inaccurate. Taking together our own field observation and the literature review, we found 121 taxa consumed by $S$. ornatus. A total of 78 bird species were reported, mainly Galliformes, followed by medium-sized mammals (38 species), well represented by Rodentia and Primates.
\end{abstract}

KEY-WORDS: birds of prey, diet, nesting, predation, review.

\section{INTRODUCTION}

The Ornate Hawk-Eagle Spizaetus ornatus is a large and imposing bird of prey from the family Acciptridae. The species' size ranges from 56 to $68.5 \mathrm{~cm}$ in length (Howell \& Webb 1995) and 820 to $1272 \mathrm{~g}$ in body mass (Dunning-Jr. 2008). Ornate Hawk-Eagle occurs in rainforest, wetlands, riparian forests, seasonally deciduous and semi-deciduous forests, up to elevations of $-1800 \mathrm{~m}$ a.s.l. (Ínigo et al. 1987, del Hoyo et al. 1994, FergusonLees \& Christie 2001). Ornate Hawk-Eagle has a wide distribution, from Mexico to the northern of Argentina and Paraguay, including all Brazil (Ferguson-Lees \& Christie 2001), but it is considered rare throughout its range, mainly in southern populations such as in northern Argentina and south-southeastern Brazil (Márquez et al. 2000).

Observations of birds of prey, like the large Ornate Hawk-Eagle, are difficult to get because of the low densities, shy behavior and closed forest preferences (Bibby et al. 1992). For these reasons, basic information of its life history is still poorly known (Robinson 1994, Naveda-Rodríguez 2004).

Despite Ornate Hawk-Eagle is often found in forest fragment edges and even in open degraded areas (FergusonLees \& Christie 2001) it needs preserved habitats for breeding (Lyon \& Kuhnigk 1985). The combination of habitat loss and low breeding rate has been threatening of extinction this species, with the populations decreasing throughout its occurrence area (Márquez et al. 2000). It is not a surprise that Ornate Hawk-Eagle is globally "Near Threatened" (BirdLife International 2016), and has been listed as threatened in several locations, such as Paraguay (del Castillo \& Clay 2005). In Brazil, S. ornatus is "Critically Endangered" in the states of Rio Grande do Sul (Rio Grande do Sul 2014), São Paulo (Silveira et al. 2009), Espírito Santo (Simon et al. 2007) and Santa Catarina (CONSEMA 2011), and "Endangered" in Paraná (Mikich \& Bérnils 2004), Rio de Janeiro (Alves et al. 2000) and Minas Gerais (Copam 2010).

The overall negative impacts of habitat changes in the distribution and in the reproduction of the species also affect feeding behavior. It is known that Ornate Hawk-Eagle hunts its prey in roosts on dense vegetation, including the edge and the interior of forests patches (Robinson 1994). Ornate Hawk-Eagle has fast flying attack to reach and capture the prey both in trees and on the ground (Trail 1987, Ferguson-Lees \& Christie 2001). Prey include bird flocks such as egrets in nesting colonies (Hilty 2003), birds that feed in flocks (Kilham 1978, Robinson 1994), groups of birds in reproductive display such as Guianan Cock-of-the-Rock (Rupicola rupicola), and also mammals such as monkey groups (Hilty 2003). The literature shows that most of the food items of $S$. 
ornatus consists of medium-sized mammals and large birds, which are frequently missing in forest due to the defaunation caused by human-induced fragmentation (Kurten 2013); and less frequently, reptiles (Klein et al. 1988). However, the published data is scarce and there are few studies with more detailed data (e.g., Lyon \& Kuhnigk 1985 and Madrid et al. 1992 in Guatemala, Klein et al. 1988 in Brazil, Clinton-Eitniear et al. 1991 in Belize, and Robinson 1994 in Peru). Understanding the diet of $S$. ornatus allow us to better planning actions for Ornate Hawk-Eagle conservation.

This study shows a new predation record by a young $S$. ornatus observed at the field. In addition, a data set was collected from the nest of Ornate Hawk-Eagle. Finally, we reviewed dietary records from published articles and unpublished data shared between researchers about Ornate Hawk-Eagle.

\section{METHODS}

\section{Field observations}

Observations and recordings were carried out in the Conceição do Mato Dentro, southeast Minas Gerais state, Brazil. The area is inserted in an ecotone between areas of Atlantic Forest and Cerrado Domains, located in the eastern portion of the Espinhaço Range (18 ${ }^{\circ} 54.584^{\prime} \mathrm{S}$; $\left.43^{\circ} 25.596^{\prime} \mathrm{W}\right)$. The Atlantic Forest and the Cerrado are considered hotspots of biodiversity (Mittermeier et al. 2004), reflected in bird diversity, which have the highest number of threatened (54 species in the Atlantic Forest and 14 in the Cerrado) (Marini \& Garcia 2005) and endemic species in Brazil (223 species in the Atlantic Forest and 29 in the Cerrado region) (Silva 1997).

Observations were carried out in semi-deciduous riparian forest areas and rocky outcrops at $816 \mathrm{~m}$ altitude. Because of the recent fragmentation, the area is a mosaic between forest fragments and open areas. In 07 April 2016, we observed a young S. ornatus resting on the ground in an open area, near the forest remnant. Two days later, an individual with adult plumage was found flying in thermals. Since then, those individuals were monitored to identify prey captured by the species. Through observations and monitoring the young $S$. ornatus, we discovered, at the same month, the nest between thick forks of Jatobá tree (Hymenaea courbari) approximately $25 \mathrm{~m}$ height and about $300 \mathrm{~m}$ from the predation site.

On 01 June 2016 the tree nest was cut down (properly authorized by the competent organs), and we collected the nest with its content. At this moment the young had already abandoned the nest and its withdrawal could be done without causing direct damage to the birds. All biological material contained in the nest (bones and feathers) was screened and identified with the help of specialists from the Natural History Museum of the Catholic University of Minas Gerais.

\section{Literature review}

To review the available information of the diet of Ornate Hawk-Eagle, we consulted scientific articles (searched in Web of Science, Scirus, JSTOR and Scielo), unpublished information, and photographs from citizen science webpages (e.g., http://www.wikiaves.com.br/, http://ibc. lynxeds.com/, http://www.ebird.org). In these cases, authors were contacted to confirm information.

\section{RESULTS}

\section{Field observation}

On 07 April 2016, a young of S. ornatus was observed among the branches of a cut tree on the floor feeding an adult Lesser Yellow-headed Vulture (Cathartes burrovianus) (Fig. 1). On the next day the same bird was observed again feeding on the carcass in the same place, consuming all flesh and bones, leaving feathers.

In the following days, new predation records were observed. The same young was observed feeding on the rest of a mammal and at least two small birds at the same location of the previous record. However, it was not possible to identify species. In these latter cases the bird was feeding in the forest edge, between canopy branches.

The nest material collected revealed a higher consumption of birds. Five species of birds were identified by feather remains (Penelope sp., Trogon surrucura, Patagioenas picazuro, Hydropsalis albicollis, Piaya cayana and Strigidae). In addition, two jaws and one pelvic

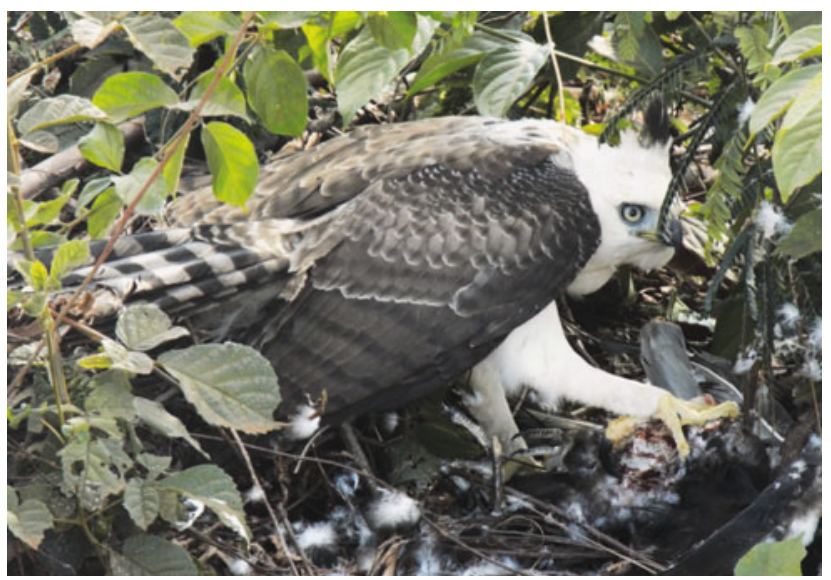

Figure 1. Ornate Hawk-Eagle (Spizaetus ornatus) eating a Lesser Yellow-headed Vulture (Cathartes burrovianus) in 07 April 2016, in Conceição do Mato Dentro, southeast of Minas Gerais, Brazil. Photo author: Michele A. Ferreira. 
bone were identified, both attributed to two individuals, one adult and one juvenile of Paraguayan Hairy Dwarf Porcupine (Coendou spinosus).

\section{Literature review}

We gathered 23 papers, ten books, five records of unpublished information and photographs from citizen science and one conference abstract with some information on the species' diet. Most data were from observations of carcasses taken from nest, while other provided information about attacks and attempted predation events.

Taking together our own field observation and the literature review, we found 121 taxa consumed by S. ornatus (Table 1). The largest number of species ( 78 species) was birds, mainly Galliformes, followed by medium-sized mammals (38 species), well represented by Rodentia and Primates. Among the Squamata, there are few records of predation on Iguana iguana and some unidentified snakes and lizards.

\section{DISCUSSION}

\section{Field observations}

The young S. ornatus seems to feed on a dead individual, since no traces of predation were observed. Jones \& Dorward (2014) recorded the "interaction" between $S$. ornatus and a bovine femur and suggested that the species may present scavenging behavior. However, since the young bird was observed again feeding on the carcass in the consecutive days, it is possible that the vulture was captured before the first record. This would explain the absence of traces of recent predation regarding that return-hunting behavior is rare among birds of prey (e.g., Springer et al. 2011, Whitacre et al. 2012).

Detailed records of predation of S. ornatus are nonexistent or inaccurate. Stiles \& Skutch (1989) have already mentioned that the species "captures birds up size of guans or vulture", and Sigrist (2006) mention that they "catch macaws, parrots, vultures, Coragyps atratus". However, those authors did not provided details, probably referring

Table 1. Food items consumed by Spizaetus ornatus based in a literature review and based in the nest material from Minas Gerais, Brazil (current study).

\begin{tabular}{|c|c|c|c|c|}
\hline Class/Order & Family & Species & Common name & Reference \\
\hline \multicolumn{5}{|l|}{ Aves } \\
\hline \multirow[t]{10}{*}{ Tinamiformes } & Tinamidae & Crypturellus boucardi & Slaty-breasted Tinamou & Whitacre et al. 2012 \\
\hline & & $\begin{array}{l}\text { Crypturellus } \\
\text { cinnamomeus }\end{array}$ & $\begin{array}{l}\text { Eastern Thicket } \\
\text { Tinamou }\end{array}$ & Whitacre et al. 2012 \\
\hline & & Crypturellus obsoletus & Brown Tinamou & Joenck et al. 2011 \\
\hline & & Crypturellus parvirostris & Small-billed Tinamou & Greco et al. 2004 \\
\hline & & Crypturellus soui & Little Tinamou & Flatten et al. 1989, Whitacre et al. 2012 \\
\hline & & $\begin{array}{l}\text { Crypturellus sp. } \\
\text { (variegatus or soui) }\end{array}$ & Tinamou & Klein et al. 1988 \\
\hline & & Crypturellus sp. & Tinamou & Madrid et al. 1991, 1992 \\
\hline & & Tinamus major & Great Tinamou & $\begin{array}{l}\text { Flatten } \text { et al. 1989, Madrid et al. 1991, 1992, } \\
\text { Whitacre } \text { et al. } 2012\end{array}$ \\
\hline & & $\begin{array}{l}\text { Tinamus sp. (major or } \\
\text { guttatus) }\end{array}$ & Tinamou & Klein et al. 1988 \\
\hline & & unidentified Tinamou & Tinamou & Lyon \& Kuhnigk 1985 \\
\hline Anseriformes & Anatidae & Amazonetta brasiliensis & Brazilian Teal & Greco et al. 2004 \\
\hline \multirow[t]{8}{*}{ Galliformes } & Cracidae & Crax rubra & Great Curassow & $\begin{array}{l}\text { Russell 1964, Flatten et al. 1989, Madrid et } \\
\text { al. 1992, Phillips \& Hatten 2013, Whitacre et } \\
\text { al. } 2012\end{array}$ \\
\hline & & Oreophasis derbianus & Horned Guan & Gómez-de-Silva 2006 \\
\hline & & Ortalis garrula & $\begin{array}{l}\text { Chestnut-winged } \\
\text { Chachalaca }\end{array}$ & Olrog 1985 \\
\hline & & Ortalis guttata & Speckled Chachalaca & Sigrist 2006 \\
\hline & & Ortalis motmot & Little Chachalaca & Klein et al. 1988 \\
\hline & & Ortalis ruficauda & $\begin{array}{l}\text { Rufous-vented } \\
\text { Chachalaca }\end{array}$ & Friedmann \& Smith-Jr. 1955 \\
\hline & & Ortalis vetula & Plain Chachalaca & $\begin{array}{l}\text { Lyon \& Kuhnigk 1985, Flatten et al. } 1989, \\
\text { Madrid et al. 1991, 1992, Whitacre et al. } 2012\end{array}$ \\
\hline & & Penelope obscura & Dusky-legged Guan & Joenck et al. 2011 \\
\hline
\end{tabular}




\begin{tabular}{|c|c|c|c|c|}
\hline Class/Order & Family & Species & Common name & Reference \\
\hline & & Penelope purpurascens & Crested Guan & $\begin{array}{l}\text { Kilham 1978, Lyon \& Kuhnigk 1985, Madrid } \\
\text { et al. 1992, Abadia \& Navarro 2011, Whitacre } \\
\text { et al. } 2012\end{array}$ \\
\hline & & Penelope sp. (cf. jacuacu) & Guan & Klein et al. 1988 \\
\hline & & Penelope sp. (cf. marail) & Guan & Klein et al. 1988 \\
\hline & & Penelope superciliaris & Rusty-margined Guan & Greco et al. 2004, Lucheti 2015 \\
\hline & & $\begin{array}{l}\text { Penelope sp. (cf. } \\
\text { superciliaris or obscura) }\end{array}$ & Guan & This study. \\
\hline & Odontophoridae & Odontophorus capueira & $\begin{array}{l}\text { Spot-winged Wood- } \\
\text { quail }\end{array}$ & Joenck et al. 2011 \\
\hline & & Odontophorus guttatus & Spotted Wood-quail & Whitacre et al. 2012 \\
\hline & Phasianidae & Gallus gallus & Domestic chicken & $\begin{array}{l}\text { Friedmann \& Smith-Jr. 1955, Ffrench 1991, } \\
\text { Robinson } 1994\end{array}$ \\
\hline & & Meleagris ocellata & Ocellated Turkey & $\begin{array}{l}\text { Madrid et al. 1991, 1992, Phillips \& Hatten } \\
\text { 2013, Whitacre et al. } 2012\end{array}$ \\
\hline Suliformes & Anhingidae & Anhinga anhinga & Anhinga & Greco et al. 2004 \\
\hline \multirow[t]{2}{*}{ Pelecaniformes } & Ardeidae & Ardea alba & Great White Egret & Teixeira 2015 \\
\hline & & Butorides striata & Green-backed Heron & Aldes Lamounier, pers. comm. \\
\hline Cathartiformes & Cathartidae & Coragyps atratus & American Black Vulture & Dickey \& van Rossem 1938 \\
\hline \multirow[t]{5}{*}{ Gruiformes } & Psophiidae & Psophia leucoptera & $\begin{array}{l}\text { White-winged } \\
\text { Trumpeter }\end{array}$ & Robinson 1994 \\
\hline & Rallidae & Aramides cajaneus & Grey-necked Wood-rail & Robinson 1994, Whitacre et al. 2012 \\
\hline & & Gallinula chloropus & Common Moorhen & Greco et al. 2004 \\
\hline & & Pardirallus nigricans & Blackish Rail & Greco et al. 2004 \\
\hline & & Porphyrio martinicus & Purple Gallinule & Robinson 1994 \\
\hline \multirow[t]{8}{*}{ Columbiformes } & Columbidae & Columba sp. & Pigeons & Flatten et al. 1989, Madrid et al. 1991, 1992 \\
\hline & & $\begin{array}{l}\text { Columbidae (Columba, } \\
\text { Leptotila, or Geotrygon) }\end{array}$ & Pigeons and Doves & Whitacre et al. 2012 \\
\hline & & Geotrygon montana & Ruddy Quail-Dove & Whitacre et al. 2012 \\
\hline & & Leptotila plumbeiceps & Grey-headed Dove & Lyon \& Kuhnigk 1985 \\
\hline & & Leptotila rufaxilla & Grey-fronted Dove & Joenck et al. 2011 \\
\hline & & Leptotila verreauxi & White-tipped Dove & Greco et al. 2004 \\
\hline & & Patagioenas picazuro & Picazuro Pigeon & Greco et al. 2004, this study \\
\hline & & Patagioenas speciosa & Scaled Pigeon & Whitacre et al. 2012 \\
\hline Opisthocomiformes & Opisthocomidae & Opisthocomus hoazin & Hoatzin & Dornas \& Pinheiro 2007, Robinson 1994 \\
\hline Cuculiformes & Cuculidae & Piaya cayana & $\begin{array}{l}\text { Common Squirrel- } \\
\text { Cuckoo }\end{array}$ & Joenck et al. 2011, this study \\
\hline Strigiformes & Strigidae & Megascops guatemalae & $\begin{array}{l}\text { Guatemalan Screech- } \\
\text { Owl }\end{array}$ & Madrid et al. 1992, Whitacre et al. 2012 \\
\hline Caprimulgiformes & Caprimulgidae & Hydropsalis albicollis & Pauraque & This study. \\
\hline Trogoniformes & Trogonidae & Trogon surrucura & Surucua Trogon & This study. \\
\hline Coraciiformes & Alcedinidae & Megaceryle torquata & Ringed Kingfisher & Wetmore 1965 \\
\hline \multirow[t]{6}{*}{ Piciformes } & Ramphastidae & Aulacorhynchus prasinus & Emerald Toucanet & $\begin{array}{l}\text { Abadia \& Navarro 2011, Whitacre et al. 2012, } \\
\text { Monroy-Ojeda et al. } 2014\end{array}$ \\
\hline & & Pteroglossus castanotis & Chestnut-eared Araçari & Leonardo Lopes pers. comm. \\
\hline & & Pteroglossus torquatus & Collared Araçari & $\begin{array}{l}\text { Madrid et al. 1991, Phillips \& Hatten 2013, } \\
\text { Whitacre et al. } 2012\end{array}$ \\
\hline & & Ramphastos cuvieri & Cuvier's Toucan & Giudice 2007 \\
\hline & & Ramphastos sulfuratus & Keel-billed Toucan & $\begin{array}{l}\text { Flatten et al. 1989, Madrid et al. 1991, 1992, } \\
\text { Phillips \& Hatten 2013, Whitacre et al. } 2012\end{array}$ \\
\hline & & Ramphastos vitellinus & Channel-billed Toucan & Klein et al. 1988 \\
\hline
\end{tabular}




\begin{tabular}{|c|c|c|c|c|}
\hline Class/Order & Family & Species & Common name & Reference \\
\hline & Picidae & $\begin{array}{l}\text { Campephilus } \\
\text { guatemalensis }\end{array}$ & Pale-billed Woodpecker & Whitacre et al. 2012 \\
\hline & & Melanerpes sp. & Woodpecker & Madrid et al. 1991 \\
\hline \multirow[t]{10}{*}{ Psittaciformes } & Psittacidae & Amazona autumnalis & Red-lored Amazon & Whitacre et al. 2012 \\
\hline & & Amazona farinosa & Southern Mealy Amazon & Whitacre et al. 2012 \\
\hline & & $\begin{array}{l}\text { Amazona sp. (cf. } \\
\text { albifrons) }\end{array}$ & $\begin{array}{l}\text { Probably White-fronted } \\
\text { Amazon }\end{array}$ & Whitacre et al. 2012 \\
\hline & & Ara macao & Scarlet Macaw & Klein et al. 1988 \\
\hline & & Ara sp. & Macaw & Robinson 1994 \\
\hline & & $\begin{array}{l}\text { Ara sp. (cf. } \\
\text { Orthopsittaca manilatus) }\end{array}$ & Red-bellied Macaw & Klein et al. 1988 \\
\hline & & Aratinga sp. & Parakeet & Robinson 1994 \\
\hline & & Brotogeris sp. & Parakeet & Robinson 1994 \\
\hline & & Pionus sp. & Parrot & Naveda-Rodríguez 2004 \\
\hline & & $\begin{array}{l}\text { Psittacidae (Pionus or } \\
\text { Pionopsitta) }\end{array}$ & Parrot & Whitacre et al. 2012 \\
\hline \multirow[t]{9}{*}{ Passeriformes } & Bombycillidae & Ptilogonys caudatus & $\begin{array}{l}\text { Long-tailed Silky- } \\
\text { Flycatcher }\end{array}$ & Acosta-Chaves et al. 2012 \\
\hline & Cotingidae & Rupicola rupicola & $\begin{array}{l}\text { Guianan Cock-of-the- } \\
\text { Rock }\end{array}$ & Trail 1987 \\
\hline & Tyrannidae & Tyrannus melancholicus & Tropical Kingbird & Souza 2013 \\
\hline & Corvidae & Cyanocorax cyanopogon & White-naped Jay & Luiz Trinchão pers. comm. \\
\hline & & Psilorhinus morio & Brown Jay & $\begin{array}{l}\text { Whitacre et al. 2012, Flatten et al. 1989, } \\
\text { Madrid et al. } 1992\end{array}$ \\
\hline & Icteridae & Cacicus cela & Yellow-rumped Cacique & Robinson 1994 \\
\hline & & Dives dives & Melodious Blackbird & Whitacre et al. 2012 \\
\hline & Icteridae & Psarocolius montezuma & Montezuma Oropendola & Whitacre et al. 2012 \\
\hline & Undetermined & $\begin{array}{l}\text { Probably Quiscalus } \\
\text { mexicanus or } \\
\text { Crotophaga sulcirostris }\end{array}$ & $\begin{array}{l}\text { Great-tailed Grackle or } \\
\text { Groove-billed Ani }\end{array}$ & Whitacre et al. 2012 \\
\hline \multicolumn{5}{|l|}{ Mammalia } \\
\hline Carnivora & Procyonidae & Nasua narica & White-nosed Coati & $\begin{array}{l}\text { Madrid et al. 1991, 1992, Abadia \& Navarro } \\
\text { 2011, Whitacre et al. 2012 }\end{array}$ \\
\hline \multirow[t]{5}{*}{ Chiroptera } & Phyllostomidae & Artibeus jamaicensis & $\begin{array}{l}\text { Jamaican Fruit-eating } \\
\text { Bat }\end{array}$ & Whitacre et al. 2012 \\
\hline & & Artibeus sp. & Fruit-eating Bat & Whitacre et al. 2012 \\
\hline & & Unidentified Bats & Bat & Lyon \& Kuhnigk 1985 \\
\hline & undetermined & Unidentified Bats & Bat & Madrid et al. 1991 \\
\hline & & Unidentified Bats & Bat & Lyon \& Kuhnigk 1985, Madrid et al. 1992 \\
\hline \multirow[t]{6}{*}{ Didelphimorphia } & Didelphidae & Caluromys derbianus & $\begin{array}{l}\text { Central American } \\
\text { Woolly Opossum }\end{array}$ & Madrid et al. 1992 \\
\hline & & Didelphis marsupialis & Common Opossum & Klein et al. 1988 \\
\hline & & Didelphis sp. & Opossum & Abadia \& Navarro 2011 \\
\hline & & Marmosa mexicana & $\begin{array}{l}\text { Mexican Mouse } \\
\text { Opossum }\end{array}$ & Whitacre et al. 2012 \\
\hline & & Metachirus nudicaudatus & $\begin{array}{l}\text { Brown Four-eyed } \\
\text { Opossum }\end{array}$ & Klein et al. 1988 \\
\hline & & Philander frenatus & $\begin{array}{l}\text { Southeastern Four-eyed } \\
\text { Opossum }\end{array}$ & Greco et al. 2004 \\
\hline Lagomorpha & Leporidae & Sylvilagus brasiliensis & Tapeti & Greco et al. 2004 \\
\hline \multirow[t]{2}{*}{ Pilosa } & Cyclopedidae & Cyclopes didactylus & Silky Anteater & Giudice 2007 \\
\hline & Myrmecophagidae & Tamandua mexicana & Northern Tamandua & Abadia \& Navarro 2011 \\
\hline
\end{tabular}




\begin{tabular}{|c|c|c|c|c|}
\hline Class/Order & Family & Species & Common name & Reference \\
\hline \multirow[t]{9}{*}{ Primates } & Callitrichidae & Callithrix penicillata & $\begin{array}{l}\text { Black-pencilled } \\
\text { Marmoset }\end{array}$ & Greco et al. 2004, Luiz Trinchão pers. comm. \\
\hline & & Saguinus fuscicollis & Saddleback Tamarin & Robinson 1994, Heymann 1990 \\
\hline & & Saguinus imperator & Emperor Tamarin & Terborgh 1983 \\
\hline & & Saguinus martinsi & $\begin{array}{l}\text { Martin's Ochraceous } \\
\text { Bare-face Tamarin }\end{array}$ & Cirino 2015 \\
\hline & & Saguinus mystax & Moustached Tamarin & Heymann 1990 \\
\hline & Cebidae & Saimiri boliviensis & $\begin{array}{l}\text { Bolivian/Peruvian } \\
\text { Squirrel Monkey }\end{array}$ & Boinski et al. 2003 \\
\hline & & Saimiri oerstedii & $\begin{array}{l}\text { Black-crowned Central } \\
\text { American Squirrel } \\
\text { Monkey }\end{array}$ & Boinski et al. 2003 \\
\hline & & Saimiri sciureus & $\begin{array}{l}\text { Common Squirrel } \\
\text { Monkey }\end{array}$ & Boinski et al. 2003, Lopes et al. 2015 \\
\hline & & Saimiri sp. & Squirrel Monkey & Robinson 1994 \\
\hline \multirow[t]{14}{*}{ Rodentia } & Cricetidae & Sigmodon sp. & Cotton Rat & Naveda-Rodríguez 2004 \\
\hline & Dasyproctidae & Dasyprocta azarae & Azara's Agouti & Joenck et al. 2011, Meller 2015 \\
\hline & & Dasyprocta leporina & Red-rumped Agouti & Naveda-Rodríguez 2004 \\
\hline & & Dasyprocta punctata & $\begin{array}{l}\text { Central American } \\
\text { Agouti }\end{array}$ & Whitacre et al. 2012 \\
\hline & & $\begin{array}{l}\text { Dasyproctidae } \\
\text { (Myoprocta or } \\
\text { Dasyprocta) }\end{array}$ & Agouti & Klein et al. 1988 \\
\hline & & Myoprocta sp. & Acouchi & Klein et al. 1988 \\
\hline & Echimyidae & Proechimys sp. & Spiny Rat & Robinson 1994 \\
\hline & Erethizontidae & Coendou mexicanus & $\begin{array}{l}\text { Mexican Hairy Dwarf } \\
\text { Porcupine }\end{array}$ & Whitacre et al. 2012 \\
\hline & & Coendou sp. & Porcupine & Klein et al. 1988 \\
\hline & & Coendou spinosus & Porcupine & This study. \\
\hline & Sciuridae & Sciurus granatensis & Red-tailed Squirrel & Naveda-Rodríguez 2004 \\
\hline & & Sciurus sp. & Squirrel & Madrid et al. 1991, 1992 \\
\hline & & Sciurus yucatanensis & Yucatan Squirrel & $\begin{array}{l}\text { Phillips \& Hatten 2013, Whitacre et al. 2012, } \\
\text { Flatten } \text { et al. } 1989\end{array}$ \\
\hline & Muridae & Unidentified rat & Rat & Flatten et al. 1989 \\
\hline \multicolumn{5}{|l|}{ Reptilia } \\
\hline \multirow[t]{5}{*}{ Squamata } & Colubridae & Colubridae & Unidentified snake & Robinson 1994 \\
\hline & Iguanidae & Iguana iguana & Common Green Iguana & $\begin{array}{l}\text { Clinton-Eitniear et al. 1991, Luiz Trinchão } \\
\text { pers. comm. Sidnei Dantas pers. comm. }\end{array}$ \\
\hline & Teiidae & Unidentified lizard & Lizard & Klein et al. 1988 \\
\hline & undetermined & Unidentified lizard & Lizard & Klein et al. 1988 \\
\hline & & Unidentified snake & Snake & Klein et al. 1988 \\
\hline
\end{tabular}

to Dickey \& van Rossem (1938), who, after dissecting the stomach of an adult $S$. ornatus, found rests of a monkey and fresh meat. Studying the area, the authors found a primate carcass near a Black-Vulture (Coragyps atratus) with back parts eaten. The authors then inferred that the vulture was killed by the eagle and partially eaten while feeding on the carcass of the monkey already killed a few days ago.

Among the collected material from the nest, only Penelope was found, species as large as the New World vultures (Dunning-Jr. 2008). The lack of other large bird species could be because $S$. ornatus does not carry large prey, eating them directly on the ground (Whitacre et al. 2012). Thus, to the best of our knowledge, vulture could be considerate an unusual prey of the Ornate Hawk-Eagle and eating the carcass suggests food habitat changes.

\section{Literature review and collected nest information}

Ornate Hawk-Eagle is considered an opportunistic predator, capturing prey according to availability in the environment (Clinton-Eitniear et al. 1991). However, 
in Guatemala, even in areas where large lizards are abundant, during seven years of study, there were no cases of predation of these species, indicating that $S$. ornatus is more specialist than thought (Whitacre et al. 2012).

In Manaus, Brazil, the consumption of 45 different prey was observed, of which $63.3 \%$ were identified as birds, $32.7 \%$ were mammals and only $4.1 \%$ were reptiles (Klein et al. 1988). Madrid et al. (1992) observed 83 prey in six nests, $38.5 \%$ mammals and $38.5 \%$ birds. In Guatemala, among 52 prey observed $40.4 \%$ were birds, $46.1 \%$ were mammals and $13.5 \%$ were not identified (Flatten et al. 1989). The same authors observed a different proportion of prey items in the diet of Ornate HawkEagle years later: of the 325 items identified, 56.3\% were birds (69.8\% of the biomass) and $43.7 \%$ were mammals (30.2\% of the biomass) (Whitacre et al. 2012). Among the items consumed, a predominance of large birds and medium-sized mammals was observed. Among birds, the most captured species was Cracidae (genus Ortalis and Penelope), Tinamidae and Ramphastidae (Ramphastos); among mammals, the medium rodents (Sciurus and Dasyprocta) predominated (Klein et al. 1988, Whitacre et al. 2012).

The preference by large birds and medium-sized mammals needs more attention. Deforestation could have indirect effects on Ornate Hawk-Eagle food diet, like the feeding on domestic animals such as chickens (Friedmann \& Smith-Jr. 1955, Ffrench 1991, Robinson 1994).

It should be noted, however, that most reports on predation were from carcasses found in the nest. Thus, large prey consumed in the soil or small animals are possibly underestimated. According to Bednarz (1988), diet records based in nest material are biased towards larger prey items because smaller items are more likely to be distorted or fragmented beyond recognition, and are more difficult to find than larger bones.

The knowledge about the ecological behavior of this species is still scarce. In recent years the Ornate HawkEagle populations have been declining in several places throughout its distribution (BirdLife International 2016). Among the species of Spizaetus genus, S. ornatus is one of the most affected by habitat losses and hunting leading to local extinctions (Canuto 2008). For Ornate HawkEagle, conservation efforts should take into account food items used by birds. As demonstrated in the present survey, detailed records are non-existent or inaccurate and careful must be taken when using this information.

\section{ACKNOWLEDGEMENTS}

We are grateful to Aryanne Clyvia, Cristiane Casar, Flávia Nunes, Claudia Costa, Marcelo Ferreira Vasconcelos,
Bruno Garzon, Giancarlo Zorzin, André Hirsch, Fabiano Melo and Anthony Rylands for sample identification. We thank Karla Leal, Ana Paula Otoni and Willian Menq for useful feedbacks, discussion and manuscript revisions. Bruna Horta, Marcos Anjos and Luiz Otávio de Oliveira helped with field surveys. We thank Jamie Holfeltz and Renzo Giudice for data access. We are grateful to many volunteers for providing recorded information.

\section{REFERENCES}

Abadia E.O. \& Navarro C.J. 2011. Monitoramento de Spizaetus ornatus e outras aves de rapina na reserva particular "Arroyo Negro", México. Spizaetus 11: 2-5.

Acosta-Chaves V., Granados-Rodríguez F. \& Araya-Huertas D. 2012. Predation of Long-tailed Silky Flycatcher (Ptilogonys caudatus) by Ornate Hawk-Eagle (Spizaetus ornatus) in a cloud forest of Costa Rica. Revista Brasileira de Ornitologia 20: 451-452.

Alves M.A.S., Pacheco J.F., Gonzaga L.A.P., Cavalcanti R.B., Raposo M.A., Yamashita C., Maciel N.C. \& Castanheira M. 2000. Aves, p. 113-124. In: Bergallo H.G., Rocha C.F.D., Alves M.A.S. \& van Sluys M. (eds.). A fauna ameaçada de extinção do estado do Rio de Janeiro. Rio de Janeiro: Editora da UERJ.

Bednarz J.C. 1988. Comparative study of the breeding ecology of Harris' and Swainson's Hawks in southeastern New Mexico. Condor 90: 311-323.

Bibby C.J., Burges N.D. \& Hill D.A. 1992. Bird census techniques. London: Academic Press.

BirdLife International. 2016. Spizaetus ornatus. The IUCN red list of threatened species. http://dx.doi.org/10.2305/IUCN.UK.2012-1. RLTS.T22696197A40380758.en (Access on 13 November 2018).

Boinski S., Kauffman L., Westoll A., Stickler C.M. Cropp S. \& Ehmke E. 2003. Are vigilance, risk from avian predators and group size consequences of habitat structure? A comparison of three species of Squirrel Monkey (Saimiri oerstedii, S. boliviensis, and S. sciureus). Behaviour 140: 1421-1467.

Canuto M. 2008. Observations of two Hawk-Eagle species in a humid lowland Tropical Forest reserve in central Panama. Journal of Raptor Research 42: 287-292.

Cirino B. 2015. WA1958085, Spizaetus ornatus (Daudin, 1800). http://www.wikiaves.com/1958085 (Access on 16 May 2016).

Clinton-Eitniear J., Gartside M.R. \& Kainer M.A. 1991. Ornate Hawk-Eagle feeding on Green Iguana. Journal of Raptor Research 25: 19-20.

CONSEMA [Conselho Estadual do Meio Ambiente]. 2011. Resolução $N^{o}$. 002/2011. http://www.fatma.sc.gov.br/upload/Fauna/ resolucao_fauna_002_11_fauna.pdf (Access on 16 April 2019).

COPAM [Conselho Estadual de Política Ambiental]. 2010. Deliberação Normativa $N^{o}$. 147/2010. http://www.siam.mg.gov. $\mathrm{br} / \mathrm{sla} /$ download.pdf?idNorma=13192 (Access on 16 April 2019).

del Castillo H. \& Clay R.P. 2005. Atlas de las aves del Paraguay. Asunción: Guyra Paraguay.

del Hoyo J., Elliot A. \& J. Sargatal. 1994. Handbook of the birds of the world, v. 2 (New World vultures to Guineafowl). Barcelona: Lynx Editions.

Dickey D.R. \& van Rossem A.J. 1938. The birds of El Salvador. Field Museum of Natural History, Zoological Series 23: 5-601.

Dornas T. \& Pinheiro R.T. 2007. Predação de Opisthocomus hoazin por Spizaetus ornatus e de Bubulcus ibis por Bubo virginianus em Tocantins, Brasil. Revista Brasileira de Ornitologia 15: 601-604.

Dunning-Jr. J.B. 2008. Handbook of avian body masses. Boca Raton: CRC Press. 
Ferguson-Lees J. \& Christie D.A. 2001. Raptors of the world. Boston: Houghton Mifflin Company.

Ffrench R. 1991. A guide to the birds of Trinidad and Tobago. Ithaca: Comstock Publishing Associates.

Flatten C.J., Madrid J.A., Hernandez A. \& Gerhardt R. 1989. Observations at the nest of an Ornate Hawk-Eagle, p. 69-79. In: Burnham W.A., Jenny J.P. \& Turtley C.W. (eds.). Maya Project Progress Report II. Boise: The Peregrine Fund, Inc.

Friedmann H. \& Smith-Jr. F.D. 1955. A further contribution to the ornithology of northeastern Venezuela. Proceedings of the United States National Museum 104: 463-524.

Giudice R. 2007. First nesting report of the Ornate Hawk-Eagle (Spizaetus ornatus) in Peru, p. 9-13. In: Bildstein K.L., Barber D.R. \& Zimmerman A. (eds.). Neotropical raptors. Orwigsburg: Hawk Mountain Sanctuary.

Gómez-de-Silva H. 2006. Mexico. North American Birds 60: 444-451.

Greco M.V., Andrade M.A., Carvalho G.D.M., Carvalho-Filho E.P.M. \& Carvalho C.E. 2004. Callithrix penicillata na dieta de Spizaetus ornatus (Aves: Accipitridae) em área de Cerrado no estado de Minas Gerais, p. 155-160. In: Mendes S.L. \& Chiarello A.G. (eds.). A primatologia no Brasil, v. 8. São Paulo: Sociedade Brasileira de Primatologia.

Heymann E.W. 1990. Reactions of wild tamarins, Saguinus mystax and Saguinus fuscicollis, to avian predators. International Journal of Primatology 11: 327-337.

Hilty S.L. 2003. Birds of Venezuela. Princeton: Princeton University Press.

Howell S.N.G. \& Webb S. 1995. A guide to the birds of Mexico and northern Central America. Oxford: Oxford University Press.

Ínigo E.E., Ramos M. \& González F. 1987. Two recent records of Neotropical eagles in southern Veracruz, México. Condor 89: 671-672.

Joenck C.M., Zilio F. \& Mendonça-Lima A. 2011. First record of breeding of the Ornate Hawk-Eagle (Spizaetus ornatus) in southern Brazil. Hornero 26: 163-166.

Jones S.E.I. \& Dorward L.J. 2014. Possible scavenging behaviour in Ornate Hawk-Eagle Spizaetus ornatus in Amazonas, Brazil. Revista Brasileira de Ornitologia 22: 27-31.

Kilham L. 1978. Alarm call of Crested Guan when attacked by Ornate Hawk-Eagle. Condor 80: 347-348.

Klein B.C., Harper L.H., Bierragaard R.O. \& Powell G.V.N. 1988. The nesting and feeding behavior of the Ornate Hawk-Eagle near Manaus, Brazil. Condor 90: 239-241.

Kurten E.L. 2013. Cascading effects of contemporaneous defaunation on Tropical Forest communities. Biological Conservation 163: $22-32$.

Lopes G.P., Guimarães D.P. \& Jaskulski A. 2015. Predation of Saimiri cassiquiarensis (Lesson, 1840) (Primates: Cebidae) by Spizaetus ornatus (Daudin, 1800) (Accipitriformes: Accipitridae) in the Brazilian Amazon. Atualidades Ornitológicas 186: 20.

Lucheti W.D. 2015. WA2083911, Spizaetus ornatus (Daudin, 1800). http://www.wikiaves.com/2083911 (Access on 16 May 2016).

Lyon B. \& Kuhnigk A. 1985. Observations on nesting Ornate HawkEagles in Guatemala. Wilson Bulletin 97: 141-147.

Madrid J.A., Madrid H.D., Funes S.H., Avila J.A., Botzoc R.G. \& Ramos A. 1991. Reproductive biology and behavior of Ornate Hawk-Eagle in Tikal National Park, p. 92-113. In: Whitacre D.F., Burnham W.A. \& Jenny J.P. (eds.). Maya Project Progress Report IV. Boise: The Peregrine Fund Inc.

Madrid H.D., Madrid R.A., Echeverría J.R.C., Alvarez J.S.C., Rivera M.C., Avila W.E.M. \& Cáal A.R. 1992. Behavior and breeding biology of the Ornate Hawk-Eagle, p. 179-191. In: Whitacre D.F. \& Thorstrom R.K. (eds.). Maya Project Progress Report V. Boise: The Peregrine Fund Inc.

Marini M.A. \& Garcia F.I. 2005. Bird conservation in Brazil. Conservation Biology 19: 665-671.

Márquez R.C., Zalles J., Stubbs L. \& Bildstein K.E.S. 2000. Latin American Falconiformes Conservation Assessment and Management Plan. Apple Valley: IUCN.

Meller D.A. 2015. WA1703770, Spizaetus ornatus (Daudin, 1800). http://www.wikiaves.com/1703770 (Access on 14 April 2016).

Mikich S.B. \& Bérnils R.S. 2004. Livro vermelho da fauna ameaçada no estado do Paraná. Curitiba: Instituto Ambiental do Paraná.

Mittermeier R.A., Gil P.R., Hoffmann M., Pilgrim J., Brooks T., Mittermeier C.G., Lamoreux J. \& Fonseca G.A.B. 2004. Hotspots revisited: earth's biologically richest and most endangered ecoregions. Mexico City: CEMEX.

Monroy-Ojeda A., Gibert-Isern S. \& Orantes-Abadía E. 2014. Monitoramento do sucesso reprodutivo do Gavião-de-Penacho (Spizaetus ornatus) em Arroyo Negro, Chiapas: gerando um estudo pioneiro de aves de rapina Neotropicais no México. Spizaetus 18: $2-7$.

Naveda-Rodríguez A. 2004. Contribution to the natural history of Ornate Hawk-Eagle Spizaetus ornatus (Daudin, 1801) and Great Black-Hawk Buteogallus urubitinga (Gmelin, 1788). Revista de Ecología Latinoamerica 11: 23-26.

Olrog C.C. 1985. Status of wet forest raptors in northern Argentina, p. 191-197. In: Newton I. \& Chancellor R.D. (eds.). Conservation studies on raptors. Cambridge: ICBP Technical Publication.

Phillips R.A. \& Hatten C.J. 2013. Nest observations on the Ornate Hawk-Eagle (Spizaetus ornatus) in Belize, Central America. Boletín SAO 22: 1-9.

Rio Grande do Sul. 2014. Decreto No 51.797 de 08 de Setembro de 2014. http://www.al.rs.gov.br/filerepository/repLegis/arquivos/ DEC\%2051.797.pdf (Access on 18 April 2019).

Robinson S.K. 1994. Habitat selection and foraging ecology of raptors in Amazonian Peru. Biotropica 26: 443-458.

Russell S.M. 1964. A distributional study of the birds of British Honduras. Ornithological Monographs 1: 1-195.

Sigrist T. 2006. Aves do Brasil: uma visão artística. São Paulo: Avisbrasilis.

Silva J.M.C. 1997. Endemic bird species and conservation in the Cerrado region, South America. Biodiversity and Conservation 6: 435-450.

Silveira L.F., Benedicto G.A., Schunck F. \& Sugieda A.M. 2009. Aves, p. 87-284. In: Bressan P.M., Kierulff M.C.M. \& Sugieda A.M. (eds.). Fauna ameaçada de extinção no estado de Sáo Paulo: vertebrados. São Paulo: Fundação Parque Zoológico de São Paulo.

Simon J.E., Antas P.T.Z., Pacheco J.F., Efe M.A., Ribon R., Raposo M.A., Laps R.R., Musso C., Passamani J.A. \& Paccagnella S.G. 2007. As aves ameaçadas de extinção no estado do Espírito Santo, p. 47-64. In: Mendes S.L., Passamani M. (eds.). Livro vermelho das espécies da fauna ameaçada de extinçấo no estado do Espírito Santo. Vitória: Instituto de Pesquisas da Mata Atlântica.

Souza J.S. 2013. WA863163, Spizaetus ornatus (Daudin, 1800). http://www.wikiaves.com/863163 (Access on 16 May 2016).

Springer M.T., Nielsen C.K., Carver A.D. \& Correa N.J. 2011. Harpy Eagle (Harpia harpyja) feeding behavior on a Brown-throated Three-toed Sloth (Bradypus variegates). Journal of Raptor Research 45: $100-103$.

Stiles G. \& Skutch A.F. 1989. A guide to the birds of Costa Rica. Ithaca: Cornell University Press. 
Teixeira S.A. 2015. WA1932260, Spizaetus ornatus (Daudin, 1800). http://www.wikiaves.com/1932260 (Access on 16 May 2016).

Terborgh J. 1983. Five New World primates: a study in comparative ecology. Princeton: Princeton University Press.

Trail P.W. 1987. Predation and antipredator behavior at Guianan Cock-of-the-Rock leks. Auk 104: 496-507.

Wetmore A. 1965. The birds of the Republic of Panamá: Tinamidae (tinamous) to Rynchopidae (skimmers), Part 1. Washington:
Smithsonian Institution Press.

Whitacre D.F., Madrid J.A., Madrid H.D., Cruz R., Flatten C.J. \& Funes S.H. 2012. Ornate Hawk-Eagle, p. 203-233. In: Whitacre D.F. (ed.). Neotropical birds of prey: biology and ecology of a forest raptor community. Ithaca: Cornell University Press.

Associate Editor: Cristiano S. Azevedo. 